\title{
Zusammenhang der Leseverständnisleistungen mit sozialen Herkunftsfaktoren bei SchülerInnen der dritten Schulstufe
}

\author{
Katharina Maitz • Lisa Paleczek • Susanne Seifert • \\ Barbara Gasteiger-Klicpera
}

Online publiziert: 2. Februar 2018

(C) Der/die Autor(en) 2018. Dieser Artikel ist eine Open-Access-Publikation.

Zusammenfassung In der Grundschule erworbene Lesefähigkeiten sind die Basis für die Teilhabe an weiterführender Bildung. In der vorliegenden Studie wurden die Zusammenhänge zwischen Leseverständnis und Herkunftsfaktoren bzw. Erstsprache untersucht ( $N=778$ DrittklässlerInnen). Die Daten wurden mittels standardisierter Lesetests und Eltern- sowie Lehrendenfragebögen erhoben. Es wurde gezeigt, dass Kinder mit einer anderen Erstsprache als Deutsch in ihrem Leseverständnis im Nachteil sind, diese Leistungsunterschiede jedoch zum Teil auf Unterschiede in den sozioökonomischen Faktoren und in der Ausstattung mit kulturellen Ressourcen zurückgeführt werden konnten.

Schlüsselwörter Leseverständnisleistung · Grundschule · Sozioökonomischer Hintergrund · Kulturelle Ressourcen

\footnotetext{
K. Maitz, BA. BA. MA. · Dipl. Sprachwiss. S. Seifert, PhD · Univ. Prof. Dr. B. Gasteiger-Klicpera Institut für Erziehungs- und Bildungswissenschaft, Arbeitsbereich Integrationspädagogik und Heilpädagogische Psychologie, Universität Graz, Graz, Österreich

K. Maitz, BA. BA. MA.

E-Mail: katharina.maitz@uni-graz.at

Dipl. Sprachwiss. S. Seifert, PhD

E-Mail: susanne.seifert@uni-graz.at

Univ. Prof. Dr. B. Gasteiger-Klicpera

E-Mail: barbara.gasteiger@uni-graz.at

Mag. Mestre L. Paleczek, PhD ( $\bowtie)$

Kirchliche Pädagogischen Hochschule Graz, Graz, Österreich

E-Mail: lisa.paleczek@kphgraz.at
} 


\title{
Socioeconomic background, cultural capital, and reading comprehension in third grade children
}

\begin{abstract}
Reading comprehension abilities are an important basis for social and educational participation. The present study examined the relationship between reading comprehension abilities, first language, and other background characteristics ( $N=778$ 3rd graders). Standardized reading tests as well as parents' and teachers' questionnaires were used to collect the data. The results revealed that children whose first language was not German showed poorer reading comprehension performance than their peers with German as a first language. Differences in socioeconomic background and cultural capital partially accounted for this performance gap.
\end{abstract}

Keywords Reading comprehension $\cdot$ Primary school $\cdot$ Socioeconomic background · Cultural capital

\section{Lesekompetenz und Leseverständnisleistungen österreichischer SchülerInnen}

Lesekompetenz umfasst neben der Fähigkeit zum Entziffern von Buchstaben, Wörtern und Sätzen auch die Fähigkeit, den Inhalt, die Bedeutung eines geschriebenen Wortes, Satzes oder Textes zu verstehen und wiederzugeben. Diese Fähigkeit wird als Leseverständnis bezeichnet (vgl. Perfetti et al. 2005, S. 227 f.; Lenhard 2013, S. 46). Voraussetzungen für das Leseverständnis sind die Dekodierfähigkeit (das schnelle und richtige Erfassen von Buchstaben, Wortteilen und Wörtern) und grundlegende Elemente der linguistischen Kompetenz im Sinne von Wortschatz, Syntax und Sprachverständnis (vgl. Melby-Lervåg und Lervåg 2014, S. 409). In der Regel werden basale Leseverständnisfähigkeiten in der Grundschule erworben (vgl. McElvany et al. 2009, S. 122). Ein erfolgreicher Erwerb der Lesefähigkeiten in diesem Zeitraum ist vor allem deshalb wichtig, da hier der Grundstein sowohl für die weitere schulische als auch für die berufliche Bildung gelegt wird (vgl. Breit et al. 2016, S. 35). Leseverständnisfähigkeiten sind nicht nur dort, wo es gilt, sich über geschriebenen Text Wissen anzueignen, von großer Bedeutung, sondern auch notwendig für die uneingeschränkte gesellschaftliche Teilhabe (vgl. Naumann et al. 2010, S. 24). Angesichts dieser weitreichenden Bedeutung von Leseverständnisfähigkeiten sind die Österreich-Ergebnisse von Leistungsstudien wie PISA (Programme for International Student Assessment) oder PIRLS (Progress in International Reading Literacy Study) besorgniserregend. So zeigte sich bei PISA 2015, dass die Leseverständnisleistungen der österreichischen SchülerInnen am Ende der Pflichtschulzeit signifikant unter dem OECD-Schnitt lagen und auch unter jenen von SchülerInnen der anderen deutschsprachigen Länder (vgl. Toferer et al. 2016, S. 57 f.). Bei den 15-/ 16-jährigen österreichischen SchülerInnen sind $23 \%$ der Lese-Risikogruppe zuzuordnen, da ihre Lesekompetenzen vor allem beim Textverständnis mangelhaft sind (vgl. Toferer et al. 2016, S. 61; Breit 2016, S. 103). Die Ergebnisse von PIRLS 2011 wiederum zeigen, dass die Lesekompetenzen österreichischer SchülerInnen bereits am Ende der Grundschule vergleichsweise niedrig sind. Die Leseleistun- 
gen österreichischer SchülerInnen der vierten Schulstufe lagen hier hinter jenen von SchülerInnen aus 14 ausgewählten, strukturell ähnlichen Ländern (vgl. Suchán 2012, S. 13). Zudem wurde deutlich, dass rund $20 \%$ der SchülerInnen nur über rudimentäre Lesekompetenzen verfügten. Auch hier liegt Österreich bei den ausgewählten Vergleichsländern auf einem der letzten Plätze (vgl. Bergmüller und Wintersteller 2012, S. 14 f.). Bemerkenswert ist außerdem, dass österreichische SchülerInnen bei anspruchsvolleren Leseverständnisaufgaben deutlich größere Schwierigkeiten haben als SchülerInnen der Vergleichsländer (vgl. Wallner-Paschon 2012, S. 22f.). Im Hinblick auf die vielfältigen Konsequenzen, die mangelhafte Lesekompetenzen nach sich ziehen, ist es sinnvoll, sich mit möglichen Einflüssen auf den Erwerb von Lesekompetenzen auseinanderzusetzen, damit in der Folge entsprechende Maßnahmen gesetzt und Interventionen entwickelt werden können, die allen SchülerInnen den Erwerb des Lesens ermöglichen.

\section{Einflüsse auf den Erwerb von Lesekompetenzen}

Schulischer Kompetenzerwerb ist nicht nur von individuellen kognitiven Voraussetzungen (vgl. Wang et al. 1993, S. 277) und der Unterrichtsqualität (vgl. Hattie 2009, S. 37) abhängig, sondern auch von zahlreichen Kontextfaktoren, zu deren einflussreichsten der sozioökonomische Status der Familie (ökonomische Ressourcen; berufliche Stellung der Eltern etc.), das elterliche Bildungsniveau, der Migrationshintergrund und die Ausstattung mit lernrelevanten Ressourcen zählen. Kinder von Eltern mit akademischer Ausbildung sind beim Erwerb von Lesekompetenzen gegenüber Kindern von weniger gut ausgebildeten Eltern im Vorteil (vgl. McElvany et al. 2009, S. 126f.) und haben deutlich bessere Chancen auf einen hohen Bildungsabschluss (vgl. Sirin 2005, S. 446f.; Schreiner 2012, S. 48; Dumont et al. 2014, S. 149f.).

Im Bereich der Lesekompetenzen zeigte sich sowohl bei PISA 2015 als auch bei PIRLS 2011, dass diese Faktoren in Österreich in engem Zusammenhang mit den Leseleistungen stehen. Ein niedriger sozioökonomischer Status, eine geringe Anzahl an Büchern zuhause, ein niedriger oder kein Bildungsabschluss der Eltern sowie das Vorhandensein eines Migrationshintergrunds hängen mit geringeren Leseleistungen zusammen (vgl. Salchegger et al. 2016, S. 88 ff.; Breit 2016, S. 104; Schreiner 2012, S. 48 f.; Bergmüller und Herzog-Punzenberger 2012a, S. 53). Es ist anzunehmen, dass diese Herkunftsfaktoren ihre Wirkung bereits vor Schuleintritt entfalten. Bradbury et al. (2015) konnten für Großbritannien, Kanada, Australien und die USA belegen, dass der sozioökonomische Status einer Familie sich stark auf die Vorläuferfähigkeiten des Schriftspracherwerbs bei Kindern zu Schuleintritt auswirkt. Je nach Land wurden in dieser Studie Vorläuferfähigkeiten des Lesens wie die phonologische Bewusstheit oder auch das Erkennen von Buchstaben erhoben. Kinder aus Familien mit niedrigem sozioökonomischem Status zeigten in allen vier Ländern deutlich unterdurchschnittliche Leistungen in den untersuchten Bereichen, während die Leistungen von Kindern aus Familien mit hohem sozioökonomischem Status deutlich über dem Durchschnitt lagen. Bemerkenswert ist, dass dieser Leistungsunterschied in allen untersuchten Ländern über die Jahre hinweg 
bis zum Alter von elf Jahren (zu diesem Zeitpunkt lag der Fokus vor allem auf dem Leseverständnis) bestehen blieb (vgl. Bradbury et al. 2015, S. 15 ff.). Auch für den deutschsprachigen Raum gibt es Belege, dass Herkunftsfaktoren, insbesondere der sozioökonomische Status und der Migrationshintergrund, einen deutlichen Einfluss auf die Entwicklung der Vorläuferfertigkeiten des Schriftspracherwerbs haben. So zeigte sich beispielsweise ein negativer Einfluss des Migrationshintergrunds auf die vorschulischen sprachlichen Leistungen (vgl. Niklas et al. 2010, S. 25f.). Dies ist auch dem Umstand geschuldet, dass die Erstsprache von SchülerInnen mit Migrationshintergrund sich häufig von der Unterrichtssprache unterscheidet (vgl. Salchegger et al. 2016, S. 93; Breit et al. 2016, S. 29).

In Österreich ist der Anteil der Grundschulkinder mit Migrationshintergrund, die zuhause ausschließlich Deutsch sprechen, mit rund $8 \%$ eher gering. Fast ein Viertel spricht im Alltag ausschließlich eine andere Sprache und etwas mehr als zwei Drittel sprechen Deutsch und eine andere Sprache (vgl. Vogtenhuber et al. 2016, S. 30). Demnach wachsen nur ca. $8 \%$ der österreichischen GrundschülerInnen mit Migrationshintergrund einsprachig auf, die anderen $92 \%$ wachsen mehrsprachig auf, müssen sich also neben ihrer Erstsprache auch mit der Unterrichtssprache Deutsch auseinandersetzen. Hinzu kommt, dass sich in Österreich die Umgangssprache von der Unterrichtssprache unterscheidet. Es liegt daher nahe, zunächst den bisherigen Forschungsstand zum Faktor Mehrsprachigkeit als mögliche Einflussgröße beim Erwerb von Lesekompetenzen gründlicher zu betrachten. In weiterer Folge wird auf Abkürzungen für den Status „Unterrichtssprache entspricht der Erstsprache“ (UE) und den Status ,,von der Unterrichtssprache verschiedene Erstsprache, andere Erstsprache“ (AE) zurückgegriffen.

\subsection{Mehrsprachigkeit, soziale Herkunft und Leseleistungen}

Bei Schulleistungstests schneiden SchülerInnen mit AE im Vergleich zu Gleichaltrigen mit UE sowohl in der Primar- als auch in der Sekundarstufe schlechter ab (vgl. Pareiss und Schwandtner 2013, S. 49; Bergmüller und Herzog-Punzenberger 2012b, S. 50f.). Am Ende der Grundschule erreichten in Österreich rund $67 \%$ der SchülerInnen mit UE die Bildungsstandards und konnten somit ,altersadäquate lineare und nichtlineare Texte unterschiedlicher Länge und unterschiedlicher inhaltlicher, struktureller und sprachlicher Komplexität" (Breit et al. 2016, S. 35) verstehen. Im Gegensatz dazu war dies lediglich bei rund $37 \%$ der SchülerInnen mit AE der Fall (vgl. Breit et al. 2016, S. 39). Früh auftretende Nachteile im Erwerb von Kompetenzen aufgrund einer AE können sich zudem auf den weiteren schulischen Kompetenzerwerbsprozess, wie bspw. auf mathematische Leistungen (vgl. Paetsch et al. 2015, S. 27), negativ auswirken.

Es gibt jedoch Hinweise darauf, dass der Zusammenhang zwischen Erstsprache und Schulleistung in nicht unbeträchtlichem Ausmaß durch andere Herkunftsfaktoren bedingt ist (vgl. Becker und Schubert 2006, S. $461 \mathrm{ff}$.). Kinder mit AE haben zuhause meist deutlich weniger Bücher, ihre Eltern sind weniger gut ausgebildet und gehen öfter Berufen nach, die mit niedrigem Einkommen verbunden sind, oder sind beschäftigungslos (vgl. Schnepf 2007, S. 540 ff.; McElvany et al. 2009, S. 127; Zöller und Roos 2009, S. 96). Melby-Lervåg und Lervåg (2014) haben diese Zusammen- 
hänge in einer Metaanalyse zusammengefasst, indem sie ihr Augenmerk auf Studien richteten, in denen das Leseverständnis und dessen bedingende Aspekte von Kindern mit UE und Kindern mit AE unter Berücksichtigung der Art des Testkonstrukts und des sozioökonomischen Status untersucht wurden. Kinder mit UE erbrachten moderat bessere Leseverständnisleistungen als Kinder mit AE. Dieser Zusammenhang wurde sowohl durch das Sprachverständnis als auch die Dekodierfähigkeit und schließlich durch testspezifische Aspekte moderiert. Der sozioökonomische Status übte vor allem hinsichtlich der linguistischen Kompetenz in der Unterrichtssprache (Wortschatz und Sprachverständnis) einen starken moderierenden Effekt aus. Zwar lagen Kinder mit AE hinter den anderen Kindern zurück, der Unterschied war jedoch bei Kindern aus Familien mit hohem oder mittlerem sozioökonomische Status deutlich geringer als bei Kindern aus Familien mit niedrigem (vgl. Melby-Lervåg und Lervåg 2014, S. 427). Von der Arbeitsgruppe um Verhoeven liegt zu diesen Zusammenhängen eine Reihe von Untersuchungen vor, so auch zum negativen Einfluss einer AE auf die Leseleistungen von GrundschülerInnen (vgl. Netten et al. 2014, S. $15 \mathrm{f}$.). An anderer Stelle konnten die AutorInnen zeigen, dass sich bestimmte Herkunftsfaktoren in Form leserelevanter Ressourcen nicht unbedingt gleichermaßen auf die Leseverständnisleistung von Kindern mit UE und AE auswirken. Anders als bei Melby-Lervåg und Lervåg wirkt sich eine gute Ausstattung mit Ressourcen wie Büchern und Tageszeitungen nur für Kinder mit UE positiv auf die Leseverständnisleistungen aus. Bei Kindern mit AE zeigte sich kein Einfluss (vgl. Netten et al. 2011, S. 421 f.). Auch für den deutschen Sprachraum kann ein Zusammenhang zwischen Erstsprache, Herkunftsfaktoren und Leseverständnisleistung belegt werden. So konnten in Österreich 30\% der Leistungsunterschiede bei PIRLS 2011 zwischen Kindern mit UE bzw. AE durch Unterschiede in den kulturellen Ressourcen (bspw. Anzahl an Büchern im Haushalt) erklärt werden (vgl. Bergmüller und Herzog-Punzenberger 2012b, S. 51).

\section{Ziele und Fragestellungen}

Da die Einflüsse auf die Leseleistungen von SchülerInnen vielfältig und komplex sind, ist es aus mehreren Gründen sinnvoll, sich diesem Bereich im Rahmen der vorliegenden Studie zu widmen. Zum einen beziehen sich die meisten deutschsprachigen Untersuchungen (insbesondere jene zu österreichischen Kindern) auf die Daten aus den Schulleistungsstudien PIRLS und PISA und somit auf Kinder, die mindestens die vierte Schulstufe besuchen. Aktuelle Untersuchungen zu jüngeren Kindern in Österreich sind rar. Zum anderen sind Lesekompetenzen für die uneingeschränkte Teilhabe am gesellschaftlichen Leben (vgl. Naumann et al. 2010, S. 24) und somit auch für die erfolgreiche Integration von Menschen mit Migrationshintergrund (vgl. Esser 2012, S. 140 f.) von solch essentieller Wichtigkeit, dass die Identifikation von benachteiligenden Faktoren, vor allem in Hinblick auf die Möglichkeit ihrer Entschärfung, notwendig ist.

Der Fokus des vorliegenden Beitrags liegt auf dem Leseverständnis. Dieses soll bezogen auf Einflüsse der sozialen Herkunft und darauf, ob Deutsch die Erstsprache des Kindes ist, untersucht werden. Ziel ist es einerseits zu überprüfen, ob die 
Ergebnisse von PIRLS zum Einfluss der Erstsprache und anderer Herkunftsfaktoren auf die Leseleistung von SchülerInnen der vierten Schulstufe (vgl. Bergmüller und Herzog-Punzenberger 2012a; Breit et al. 2016) sich bereits bei SchülerInnen der dritten Schulstufe abzeichnen. Zum anderen soll die Annahme überprüft werden, dass es Unterschiede in der Stärke des Zusammenhangs von Herkunftsfaktoren mit der Leseverständnisleistung in Abhängigkeit des sprachlichen Hintergrunds gibt. Daher wird den folgenden Forschungsfragen nachgegangen:

1. Wie stark ist ein möglicher Zusammenhang zwischen der Erstsprache (UE oder AE) und den Leseverständnisleistungen von Kindern der dritten Schulstufe?

2. Inwieweit reduziert sich dieser Zusammenhang durch den Einbezug von kulturellen und ökonomischen Herkunftsfaktoren?

3. Unterscheiden sich die Korrelationen von Leseverständnis und kulturellen sowie ökonomischen Herkunftsfaktoren zwischen Kindern mit UE und Kindern mit AE?

\section{Methode}

\subsection{Stichprobe}

Die für die Beantwortung der Forschungsfragen benötigten Daten wurden am Ende der dritten Schulstufe erhoben. Insgesamt umfasste die Stichprobe 778 Kinder aus städtischen und stadtnahen Gebieten des österreichischen Bundeslandes Steiermark. Jungen waren mit 51,5\% etwas stärker vertreten als Mädchen (48,5\%). Mit knapp $65 \%$ war der überwiegende Teil der Kinder neun Jahre alt, rund ein Fünftel war acht, ca. $10 \%$ zehn und zwei Kinder bereits elf Jahre alt. Das Durchschnittsalter lag bei 9,35 Jahren ( $S D=0,45$; Range: 8-11). Von nahezu der Hälfte der Kinder $(49,7 \%)$ wurde zuhause überwiegend eine andere Sprache als Deutsch gesprochen, wobei $97,4 \%$ der Kinder mit Migrationshintergrund zuhause überwiegend eine andere Sprache als Deutsch sprachen.

\subsection{Erhebungsinstrumente und Erhebung}

Als Instrument zur Erhebung der Leseverständnisfähigkeiten wurde der „Leseverständnistest für Erst- bis Sechstklässler“ (Elfe 1-6) von Lenhard und Schneider (2006) verwendet. Dieser wurde in Form dreier Subtests konzipiert, die Wort-, Satzund Textverständnis messen. Der Subtest zum Wortverständnis umfasst 72 Items, die in drei Minuten bearbeitet werden müssen. Die Kinder müssen jeweils aus einer Liste von vier geschriebenen Wörtern das zu einem vorgegebenen Bild passende auswählen, wobei die vorgeschlagenen Wörter sich ähneln (Silbenanzahl, Buchstaben, Laute). Der Satzverständnissubtest beinhaltet 28 Items in Form von Sätzen, bei denen jeweils mindestens ein Wort fehlt und aus einer Liste von fünf vorgeschlagenen Wörtern ergänzt werden muss. Auch hier ähneln sich die vorgeschlagenen Wörter und sind zudem immer derselben Wortart zuzuordnen. Die Kinder haben dazu wiederum drei Minuten Zeit. Der Subtest zum Textverständnis besteht aus kurzen Texten, zu denen mindestens eine Frage gestellt wird. Hier gilt es aus vier 
Antwortmöglichkeiten die richtige auszuwählen, wobei verschiedene Kompetenzen erforderlich sind. So zielen manche Aufgaben auf das Auffinden isolierter, in den Texten enthaltener Informationen, andere erfordern satzübergreifendes Lesen und wieder andere verlangen Schlussfolgerungen. Insgesamt umfasst der Textverständnissubtest 20 Items und die Kinder haben sieben Minuten Zeit. Die Erhebung fand direkt an den Schulen in Form einer Klassentestung statt.

Neben den Leseverständnisleistungen wurden auch soziodemografische Daten und solche zur Erstsprache der Kinder erhoben. Hierzu wurden Fragebögen für die Eltern und die Lehrenden entwickelt, die für jedes Kind einzeln ausgefüllt wurden. Sowohl der Eltern- als auch der Lehrendenfragebogen enthielt jeweils eine Frage zur Erstsprache des Kindes, um sicherzugehen, dass zu allen Kindern Daten vorlagen. Im Elternfragebogen wurden vor allem Daten zur sozialen Herkunft der Kinder erhoben. Die Fragen bezogen sich auf den Migrationshintergrund von Eltern und Kind (,In welchem Land wurden die folgenden Personen geboren?“ Kind, Vater, Mutter offene Frage), die Staatsbürgerschaft von Eltern und Kind, die höchste abgeschlossene Ausbildung der Eltern (,Welches ist der höchste Schulabschluss der Mutter/des Vaters?" - kein Abschluss/Pflichtschule/Lehre, Fachschule/Matura/Akademie, FH, Universität - fünf Stufen) und auf die berufliche Tätigkeit der Eltern (,,Sind folgende Personen im Moment berufstätig?“ Mutter/Vater - Ja/Nein; „Wenn ja, welchen Beruf üben folgende Personen aus?" Mutter/Vater - offene Frage). Außerdem wurden mit Hilfe der Frage „Wie viele Bücher gibt es in Ihrer Wohnung ungefähr?“ (mit den Antwortmöglichkeiten ,bis 10/11-40/41-100/über 100“) Informationen zu kulturellen Ressourcen der Familie erhoben. Die Erstsprache wurde mittels der offenen Frage „Welche Sprache wird überwiegend in der Familie gesprochen?“ erfragt. Insgesamt füllten 597 Eltern den Elternfragebogen aus, was einer Rücklaufquote von $76,7 \%$ entspricht.

\subsection{Aufbereitung der Daten und statistische Auswertung}

Die erhobenen Daten aus den Fragebögen wurden für weitere Berechnungen adaptiert, indem Indizes gebildet wurden. Zunächst wurde ein Index zum Gesamtleseverständnis als Summenscore der standardisierten Werte der Subtests Wort-, Satzund Textverständnis berechnet. Um den sozioökonomischen Status der Familie abzubilden, wurden die Angaben der Eltern zu ihrer aktuellen beruflichen Tätigkeit in ISCO-08 Werte (International Standard Classification of Occupations; International Labour Organization 2012) und in weiterer Folge in ISEI-08 Werte (International Socio-Economic Index of Occupational Status; Ganzeboom 2010) überführt. Dies gelang bei 304 Müttern und 380 Vätern, bei denen ausreichend genaue Angaben vorlagen. Die ISEI Werte von Müttern und Vätern wurden schließlich zu einem Index „HISEI“ (Highest Socio-Economic Index of Occupational Status) zusammengefasst, indem der höhere der beiden Werte in den neuen Index aufgenommen wurde. Zudem wurden Eltern berücksichtigt, die beide beschäftigungslos waren. In diesen Fällen wurde der Wert 14 vergeben, der einen Punkt unter dem niedrigsten für Österreich möglichen ISEI Wert liegt (zu diesem Vorgehen vgl. bspw. Richter 2008, S. 379). So konnten schließlich 408 Fälle berücksichtigt werden. 
Über die Anzahl der Bücher im Haushalt und die höchste abgeschlossene Ausbildung der Eltern wurden bildungsrelevante materielle und immaterielle kulturelle Ressourcen der Familie erfasst. Durch die Zusammenfassung dieser Variablen zu einem Index wurde die Variable ,kulturelle Ressourcen“ (Cronbachs $\alpha=0,743$ ) generiert. Schließlich wurden die Indizes zu kulturellen Ressourcen und HISEI zum Index „Herkunftsfaktoren“ (Cronbachs $\alpha=0,810$ ) zusammengefasst und dienten als Grundlage für weitere Berechnungen.

Um die Frage nach dem Zusammenhang zwischen Erstsprache und Leseverständnisleistung zu klären, wurden die Leistungsunterschiede zwischen Kindern mit UE und AE mithilfe von t-Tests für unabhängige Stichproben zu den einzelnen Subtests sowie zum Gesamtleseverständnis überprüft. Das Problem der Alpha-Fehler-Kumulierung ist im vorliegenden Fall vernachlässigbar, da die Irrtumswahrscheinlichkeit insgesamt deutlich unter $5 \%$ liegt.

Die vorliegende Stichprobe weist eine hierarchische Struktur (SchülerInnen in Klassen in Schulen) auf. Daher wurden die Voraussetzungen für eine Mehrebenenanalyse geprüft. Von den insgesamt 41 Schulklassen wiesen (unter Berücksichtigung vollständiger Angaben zu den Herkunftsfaktoren) jedoch lediglich 18 eine Fallzahl von mindestens zehn auf. Die Gefahr nicht interpretierbarer Signifikanzen, die bei Nichtberücksichtigung der Mehrebenenstruktur der Daten besteht, ist bei solch kleinen Fallzahlen auch bei einer Mehrebenenanalyse (vgl. Hadler 2004, S. 68 ff.; Hox 2002, S. 173 ff.) vorhanden. Aus diesem Grund wurde im vorliegenden Fall von einer mehrebenenanalytischen Auswertung abgesehen, da diese keine Vorteile gegenüber der klassischen linearen Regression gehabt hätte.

Veränderungen im Zusammenhang zwischen Erstsprache und Leseverständnisleistung bei Berücksichtigung von Herkunftsfaktoren wurden daher über die Berechnung und den Vergleich zweier linearer Regressionsmodelle ermittelt. Die Bedeutung der Herkunftsfaktoren für die Leseverständnisleistung wurde zusätzlich getrennt für Kinder mit UE und AE mithilfe einer Korrelationstabelle und Fishers Z-Werten errechnet. Für die statistischen Berechnungen wurde das Softwarepaket SPSS 22 (Statistical Package for Social Science, IBM ${ }^{\circledR}$ ) verwendet.

\section{Ergebnisse}

\subsection{Unterschiede in der Leseverständnisleistung und den Herkunftsfaktoren bei Kindern mit UE und AE}

In Tab. 1 sind die Mittelwerte und Standardabweichungen der einzelnen Subtests und des Gesamtleseverständnisses getrennt für Kinder mit UE und AE dargestellt. Außerdem sind die t-Werte zu den Mittelwertunterschieden zwischen den beiden Gruppen sowie Effektstärken wiedergegeben. Es zeigt sich, dass die Mittelwertunterschiede zwischen den beiden Gruppen sowohl in allen drei Subtests als auch beim Gesamtleseverständnis signifikant sind. In allen Bereichen schneiden SchülerInnen mit AE schlechter ab als SchülerInnen mit UE. 
Tab. 1 Unterschiede in den Leseverständnisleistungen von Kindern mit UE und AE

\begin{tabular}{|c|c|c|c|c|c|c|c|c|}
\hline & \multicolumn{2}{|l|}{$\begin{array}{l}\text { Gesamt } \\
(n=724)\end{array}$} & \multicolumn{2}{|l|}{$\begin{array}{l}\mathrm{UE} \\
(n=378)\end{array}$} & \multicolumn{2}{|l|}{$\begin{array}{l}\mathrm{AE} \\
(n=346)\end{array}$} & \multirow[t]{2}{*}{$\begin{array}{l}t \\
(d f)\end{array}$} & \multirow[t]{2}{*}{ Cohens d } \\
\hline & $\begin{array}{l}M \\
(S D)\end{array}$ & Range & $\begin{array}{l}M \\
(S D)\end{array}$ & Range & $\begin{array}{l}M \\
(S D)\end{array}$ & Range & & \\
\hline $\begin{array}{l}\text { Wortverständnis } \\
\text { (Rohwerte) }\end{array}$ & $\begin{array}{l}41,63 \\
(11,67)\end{array}$ & $13-72$ & $\begin{array}{l}44,55 \\
(12,02)\end{array}$ & $13-72$ & $\begin{array}{l}38,44 \\
(10,399)\end{array}$ & $17-69$ & $\begin{array}{l}7,328 * * \\
(722)\end{array}$ & $-0,54$ \\
\hline $\begin{array}{l}\text { Satzverständnis } \\
\text { (Rohwerte) }\end{array}$ & $\begin{array}{l}18,16 \\
(4,99)\end{array}$ & $2-28$ & $\begin{array}{l}19,53 \\
(5,00)\end{array}$ & $2-28$ & $\begin{array}{l}16,65 \\
(4,549)\end{array}$ & $3-28$ & $\begin{array}{l}8,119 * * \\
(722)\end{array}$ & $-0,60$ \\
\hline $\begin{array}{l}\text { Textverständnis } \\
\text { (Rohwerte) }\end{array}$ & $\begin{array}{l}11,88 \\
(4,61)\end{array}$ & $2-20$ & $\begin{array}{l}13,27 \\
(4,629)\end{array}$ & $2-20$ & $\begin{array}{l}10,367 \\
(4,1)\end{array}$ & $3-20$ & $\begin{array}{l}8,940 * * \\
(722)\end{array}$ & $-0,66$ \\
\hline $\begin{array}{l}\text { Gesamtlese- } \\
\text { verständnis } \\
\text { (Index) }\end{array}$ & $\begin{array}{l}0,000 \\
(2,77)\end{array}$ & $-7,83-6,33$ & $\begin{array}{l}0,8259 \\
(2,792)\end{array}$ & $-7,83-6,33$ & $\begin{array}{l}-0,9023 \\
(2,462)\end{array}$ & $-6,67-5,86$ & $\begin{array}{l}8,849 * * \\
(722)\end{array}$ & $-0,65$ \\
\hline
\end{tabular}

$* * p<0,001$

Tab. 2 Unterschiede bei den Herkunftsfaktoren von Kindern mit UE und AE

\begin{tabular}{|c|c|c|c|c|c|c|c|c|}
\hline & \multicolumn{2}{|l|}{ Gesamt } & \multicolumn{2}{|l|}{ UE } & \multicolumn{2}{|l|}{$\mathrm{AE}$} & \multirow{2}{*}{$\begin{array}{l}t \\
(d f)\end{array}$} & \multirow[t]{2}{*}{ Cohens d } \\
\hline & $\begin{array}{l}M \\
(S D)\end{array}$ & $N$ & $\begin{array}{l}M \\
(S D)\end{array}$ & $N$ & $\begin{array}{l}M \\
(S D)\end{array}$ & $N$ & & \\
\hline HISEI & $\begin{array}{l}41,58 \\
(17,10)\end{array}$ & 400 & $\begin{array}{l}49,81 \\
(15,68)\end{array}$ & 205 & $\begin{array}{l}32,93 \\
(14,00)\end{array}$ & 195 & $\begin{array}{l}11,371 * * \\
(396,39)\end{array}$ & $-1,13$ \\
\hline $\begin{array}{l}\text { Kulturelle } \\
\text { Ressourcen }\end{array}$ & $\begin{array}{l}2,63 \\
(0,83)\end{array}$ & 506 & $\begin{array}{l}2,87 \\
(0,71)\end{array}$ & 273 & $\begin{array}{l}2,35 \\
(0,88)\end{array}$ & 233 & $\begin{array}{l}7,279 * * \\
(446,25)\end{array}$ & $-0,65$ \\
\hline $\begin{array}{l}\text { Herkunftsfaktoren } \\
\text { Gesamt }\end{array}$ & $\begin{array}{l}0,263 \\
(3,16)\end{array}$ & 373 & $\begin{array}{l}1,53 \\
(2,79)\end{array}$ & 190 & $\begin{array}{l}-1,09 \\
(2,97)\end{array}$ & 183 & $\begin{array}{l}8,736 * * \\
(366)\end{array}$ & $-0,91$ \\
\hline
\end{tabular}

$* * p<0,001$

Bezüglich der Ausstattung mit kulturellen Ressourcen und des sozioökonomischen Status zeigen sich teilweise noch deutlichere Unterschiede zwischen Kindern mit UE und AE, wie aus Tab. 2 ersichtlich ist.

\subsection{Veränderungen im Zusammenhang von Erstsprache und Leseverständnisleistung bei Berücksichtigung der Herkunftsfaktoren}

Um zu überprüfen, ob der Zusammenhang des Erstsprachestatus mit der Leseverständnisleistung an Stärke verliert, wenn die erhobenen Herkunftsfaktoren miteinbezogen werden, wurden lineare Regressionen gerechnet (siehe Tab. 3). Um das Ergebnis nicht zu verfälschen, wurde die Stichprobe für beide Modelle auf jene Kinder eingeschränkt, zu denen Informationen zu allen Herkunftsfaktoren vorlagen. Dadurch reduzierte sich die Stichprobe auf 373 Fälle, von denen 190 Kinder UEStatus hatten und 183 Kinder nicht. Beide Gruppen waren demnach ähnlich groß, wie es auch in der Ausgangsstichprobe der Fall war. Das erste Regressionsmodell (Modell 1) zeigt den Zusammenhang der Variable Erstsprache (Deutsch oder eine andere Sprache) mit der Leseverständnisleistung, der mit einem Wert von $\beta=-0,341$ deutlich und statistisch signifikant $(p<0,01)$ ist. Darüber hinaus wird ersichtlich, dass allein über die Variable „UE oder AE“ 11,7\% der Varianz in der Leseverständnisleistung aufgeklärt werden können. 
Tab. 3 Lineare Regressionen zum Zusammenhang der Erstsprache (Modell 1) bzw. der Erstsprache und anderer Herkunftsfaktoren (Modell 2) mit der Leseverständnisleistung

\begin{tabular}{lllll}
\hline & $\begin{array}{l}\text { Modell } 1 \\
(n=373)\end{array}$ & Beta & $\begin{array}{l}\text { Modell } 2 \\
(n=373)\end{array}$ & Beta \\
\hline Konstante & $B(S D)$ & - & $1,859^{* *}(0,455)$ & - \\
UE oder AE & $3,118^{* *}(0,435)$ & $-0,341^{* *}$ & $-1,126(0,290)$ & $-0,199^{* *}$ \\
Herkunftsfaktoren & $-1,935(0,277)$ & - & $0,297(0,045)$ & $0,335^{* *}$ \\
- & - & & Korr. $\mathrm{R}^{2}=0,204$ & \\
& Korr. $\mathrm{R}^{2}=0,117$ & & $\mathrm{~F}=48,664^{* *}$ & \\
\hline
\end{tabular}

$* * p<0,01$

Bei Betrachtung von Modell 2 zeigt sich, dass die Varianzaufklärung durch Einbeziehen des Index' zu den Herkunftsfaktoren deutlich ansteigt. Es können 20,4\% der Varianz aufgeklärt werden. Ferner wird ersichtlich, dass der Zusammenhang der Herkunftsfaktoren mit der Leseverständnisleistung positiv ist $(\beta=0,335, p<0,01)$. Ein hoher sozioökonomischer Status gepaart mit einer großen Anzahl an Büchern im Haushalt und höheren Bildungsabschlüssen der Eltern geht dementsprechend mit besserer Leseverständnisleistung der SchülerInnen einher. Gleichzeitig zeigt sich, dass sich die Bedeutung der Erstsprache unter Berücksichtigung der Herkunftsfaktoren merklich reduziert $(\beta=-0,199, p<0,01)$. Demnach kann ein Teil des negativen Zusammenhangs von Leseverständnisleistung und AE auf Unterschiede in den Herkunftsfaktoren zurückgeführt werden.

\subsection{Die Bedeutung der Herkunftsfaktoren für die Leseverständnisleistung bei SchülerInnen mit UE und AE}

Tab. 4 bildet ab, ob sich Unterschiede in der Stärke des Zusammenhangs zwischen Leseverständnisfähigkeiten und den Herkunftsfaktoren eines Kindes in Hinblick darauf, ob seine Erstsprache Deutsch ist oder nicht, ergeben. Hierzu wurden Korrelationen jeweils für Kinder mit UE und Kinder mit AE gerechnet.

Um eine Aussage darüber treffen zu können, ob die Unterschiede zwischen den Korrelationskoeffizienten signifikant sind oder nicht, wurden zuerst Fishers Z-Werte berechnet und einer Signifikanzprüfung unterzogen. Keiner der Werte lag bei einem

Tab. 4 Pearson-Korrelation (zweiseitig) von Herkunftsfaktoren mit den Leseverständnisleistungen getrennt für Kinder UE und $\mathrm{AE}$

\begin{tabular}{llll}
\hline & \multicolumn{2}{l}{ Leseverständnisleistung } & Testgröße z \\
& UE & AE & \\
\hline HISEI & $0,255^{* *}$ & $0,179^{*}$ & 0,792 (n. s.) \\
& $(n=205)$ & $(n=195)$ & \\
Kulturelle Ressourcen & $0,248^{* *}$ & $0,341^{* *}$ & 1,136 (n. s.) \\
& $(n=273)$ & $(n=233)$ & \\
Herkunftsfaktoren gesamt & $0,297^{* *}$ & $0,316^{* *}$ & 0,201 (n. s.) \\
& $(n=190)$ & $(n=183)$ & \\
\hline
\end{tabular}

$* * p<0,01 ; * p<0,05$ 
Signifikanzniveau von $p<0,05$. Dies bedeutet, dass Unterschiede zwischen den Korrelationskoeffizienten auch rein zufällig zustande gekommen sein könnten und sich die einbezogenen Herkunftsaspekte in ihrem Zusammenhang mit den Leseverständnisfähigkeiten bei Kindern mit UE und Kindern mit AE nicht statistisch signifikant voneinander unterscheiden.

\section{Diskussion}

Ziel der vorliegenden Studie war die Untersuchung des Zusammenhangs zwischen Erstsprache und Leseverständnisleistungen bei SchülerInnen der dritten Schulstufe unter Berücksichtigung sozioökonomischer und kultureller Herkunftsfaktoren. Die Ergebnisse zeigten zunächst, dass es einen deutlichen Zusammenhang zwischen Leseverständnisleistung und Erstsprache gibt, der sich dahingehend äußerte, dass ein AE-Status mit geringeren Leistungen bei Wort-, Satz- und Textverständnis einherging. Diese Ergebnisse decken sich weitgehend mit jenen von PIRLS 2011 (Bergmüller und Herzog-Punzenberger 2012b, S. 50f.).

Zudem konnte gezeigt werden, dass die Erstsprache erhebliche Erklärungskraft für Unterschiede in der Leseverständnisleistung hat (Modell 1). In weiteren Berechnungen konnte jedoch nachgewiesen werden, dass der Zusammenhang der Erstsprache mit den Leseverständnisleistungen österreichischer GrundschülerInnen nicht isoliert betrachtet werden darf. Vielmehr konnte er zu einem beträchtlichen Teil auf unterschiedliche Voraussetzungen beim sozioökonomischen Status und in den kulturellen Ressourcen zurückgeführt werden (Modell 2). Ähnliche Ergebnisse wurden zu PIRLS 2011 berichtet, wo sich rund 46\% der Leistungsunterschiede zwischen Kindern mit und ohne Migrationshintergrund über den sozialen Hintergrund der Kinder erklären ließen (vgl. Salchegger et al. 2012, S. 79) und zumindest $30 \%$ der Leistungsunterschiede zwischen Kindern mit UE und AE über Unterschiede im kulturellen Kapital erklärt werden konnten (vgl. Bergmüller und Herzog-Punzenberger 2012b, S. 51). Da in Österreich der schulische Erfolg besonders stark von der sozialen Herkunft abhängt (vgl. Schreiner 2012, S. 48), sind Kinder mit Migrationshintergrund, die AE-Status aufweisen und hinsichtlich ihres kulturellen Kapitals schwach ausgestattet sind, besonders benachteiligt. Obwohl dies seit Längerem bekannt ist, wurden die Leistungsunterschiede aufgrund unterschiedlicher Herkunftsbedingungen in den letzten Jahren nicht geringer (vgl. Suchán et al. 2012, S. 59).

Eine Betrachtung der Korrelationen von Herkunftsfaktoren mit der Leseverständnisleistung getrennt für Kinder mit UE und $\mathrm{AE}$ lässt darauf schließen, dass keine signifikanten Unterschiede in der Stärke der Zusammenhänge vorlagen. Somit kann anhand der hier vorliegenden Daten nicht belegt werden, dass bestimmte Herkunftsfaktoren stärker mit den Leseverständnisleistungen von Kindern mit UE zusammenhängen als mit jenen von Kindern mit $\mathrm{AE}$ oder umgekehrt. Allerdings sollte für zukünftige Untersuchungen berücksichtigt werden, dass andere AutorInnen zu gegenteiligen Ergebnissen gelangten, indem sie zeigten, dass sich die Ausstattung mit leserelevanten Ressourcen für Kinder mit UE und Kinder mit AE unterschiedlich auswirkt (vgl. Netten et al. 2011, S. 422). 
$\mathrm{Zu}$ betonen ist an dieser Stelle die eingeschränkte Aussagekraft der Ergebnisse der vorliegenden Studie, die vor allem im querschnittlichen und korrelativen Charakter der Daten begründet ist. Fundierte Aussagen über einen ursächlichen Einfluss der Erstsprache sowie anderer Herkunftsfaktoren auf die Leseverständnisleistung von SchülerInnen sind mit dem gewählten Forschungsdesign nicht möglich. Dennoch konnte gezeigt werden, dass in der dritten Grundschulklasse deutliche Zusammenhänge zwischen Schulleistungen und sozialer Herkunft beobachtet werden können. Zwar spielte die Erstsprache eine wichtige Rolle für die Leistung im Leseverständnis, jedoch waren ihre Auswirkungen nicht unabhängig von anderen Herkunftsfaktoren. Eine verstärkte Sensibilisierung sowohl zukünftiger als auch praktizierender LehrerInnen für diesen Themenkomplex im Rahmen von Aus- und Weiterbildung erscheint wichtig. Zudem besteht Bedarf an konkreten Interventionsprogrammen, die sich damit auseinandersetzen, wie bestehende Ungleichheiten entschärft werden können und der Leistungsrückstand von Kindern mit schwierigen sozialen Ausgangsbedingungen vermindert oder gar ausgeglichen werden kann.

Im Hinblick auf Forschungsdesiderata in diesem Bereich würden eine differenzierte Betrachtung der (schrift-)sprachlichen Kompetenz der SchülerInnen auch in der (von der Unterrichtssprache verschiedenen) Erstsprache sowie ein genauerer Blick auf die Praktiken im Elternhaus ein tieferes Verständnis des Zusammenhangs von (Schrift-)Sprachkompetenz und sozialer Herkunft fördern. Hier wären alltagsnahe Beobachtungsstudien mit empirisch-qualitativen Forschungsdesigns hilfreich. Von einer solch differenzierten Erhebung wurde in der dieser Studie zugrundeliegenden Testung jedoch abgesehen, da der Zeitaufwand für die Bearbeitung von Tests und Fragebögen für SchülerInnen (deren Leistungen auch in weiteren Kompetenzbereichen erfasst wurden), Eltern und auch LehrerInnen in einem überschaubaren Rahmen gehalten werden musste.

Darüber hinaus wäre die Frage zu betrachten, in welchem Ausmaß sich Zusammenhänge zwischen Herkunftsfaktoren und (schrift-)sprachlicher Kompetenz bereits in der ersten Grundschulklasse manifestieren, ob sie hinsichtlich ihrer Stärke über mehrere Grundschuljahre hinweg konstant bleiben oder sich verändern und welche positiven Einflussfaktoren identifiziert werden könnten. Um die Zusammenhänge zwischen dem Leseverständnis und der Erstsprache besser zu verstehen, sollten in zukünftigen Studien Faktoren der sozialen, sprachlichen und kulturellen Herkunft verstärkt Berücksichtigung finden und ihre Interaktion über längere Zeiträume analysiert werden.

Funding Open access funding provided by University of Graz.

Open Access Dieser Artikel wird unter der Creative Commons Namensnennung 4.0 International Lizenz (http://creativecommons.org/licenses/by/4.0/deed.de) veröffentlicht, welche die Nutzung, Vervielfältigung, Bearbeitung, Verbreitung und Wiedergabe in jeglichem Medium und Format erlaubt, sofern Sie den/die ursprünglichen Autor(en) und die Quelle ordnungsgemäß nennen, einen Link zur Creative Commons Lizenz beifügen und angeben, ob Änderungen vorgenommen wurden. 


\section{Literatur}

Becker, R., \& Schubert, F. (2006). Soziale Ungleichheit von Lesekompetenzen. Eine Matching-Analyse im Längsschnitt mit Querschnittdaten von PIRLS 2001 und PISA 2000. Kölner Zeitschrift für Soziologie und Sozialpsychologie, 58, 253-284.

Bergmüller, S., \& Herzog-Punzenberger, B. (2012a). Kompetenzen und Charakteristika von Kindern mit und ohne Migrationshintergrund im Zeitvergleich. In B. Suchán, C. Wallner-Paschon, S. Bergmüller \& C. Schreiner (Hrsg.), PIRLS \& TIMSS 2011. Schülerleistungen in Lesen, Mathematik und Naturwissenschaft in der Grundschule. Erste Ergebnisse (S. 52-53). Graz: Leykam.

Bergmüller, S., \& Herzog-Punzenberger, B. (2012b). Die Lesekompetenz ein- und mehrsprachiger Kinder im Ländervergleich. In B. Suchán, C. Wallner-Paschon, S. Bergmüller \& C. Schreiner (Hrsg.), PIRLS \& TIMSS 2011. Schülerleistungen in Lesen, Mathematik und Naturwissenschaft in der Grundschule. Erste Ergebnisse (S. 50-51). Graz: Leykam.

Bergmüller, S., \& Wintersteller, A. (2012). Lesen: Verteilung der Schüler/innen auf Kompetenzstufen. In B. Suchán, C. Wallner-Paschon, S. Bergmüller \& C. Schreiner (Hrsg.), PIRLS \& TIMSS 2011. Schülerleistungen in Lesen, Mathematik und Naturwissenschaft in der Grundschule. Erste Ergebnisse (S. 14-15). Graz: Leykam.

Bradbury, B., Corak, M., Waldfogel, J., \& Washbrook, E. (2015). Too many children left behind: the U.S. achievement gap in comparative perspective. New York: Russell Sage Foundation.

Breit, S. (2016). PISA 2015: Zusammenfassung der Ergebnisse. In B. Suchán \& S. Breit (Hrsg.), PISA 2015. Grundkompetenzen am Ende der Pflichtschulzeit im internationalen Vergleich (S. 101-105). Graz: Leykam.

Breit, S., Bruneforth, M., \& Schreiner, C. (Hrsg.). (2016). Standardüberprüfung 2015. Deutsch, 4. Schulstufe. Bundesergebnisbericht. Salzburg: BIFIE.

Dumont, H., Maaz, K., Neumann, M., \& Becker, M. (2014). Soziale Ungleichheiten beim Übergang von der Grundschule in die Sekundarstufe I. Theorie, Forschungsstand, Interventions- und Fördermöglichkeiten. Zeitschrift für Erziehungswissenschaft, 17(Suppl. 24), 141-165.

Esser, H. (2012). Sprache und Integration. Eine Zusammenfassung und einige Anmerkungen. In M. Matzner (Hrsg.), Handbuch Migration und Bildung (S. 140-154). Weinheim: Beltz.

Ganzeboom, H. (2010). A new international socio-economic index (ISEI) of occupational status for the international standard classification of occupation 2008 (ISCO-08) constructed with data from the ISSP 2002-2007. Annual Conference of International Social Survey Programme, Lisbon.

Hadler, M. (2004). Die Mehrebenen-Analyse. Ihre praktische Anwendung und theoretische Annahmen. Österreichische Zeitschrift für Soziologie, 29(1), 53-74.

Hattie, J.A.C. (2009). Visible learning. A synthesis of over 800 meta-analyses relating to achievement. London, New York: Routledge.

Hox, J. (2002). Multilevel analysis. Techniques and applications. Mahwah, NJ, London: Lawrence Erlbaum Associates, Inc.

International Labour Office (2012). International standard classification of occupations: ISCO-08. Genf: ILO.

Lenhard, W. (2013). Leseverständnis und Lesekompetenz: Grundlagen - Diagnostik - Förderung. Stuttgart: Kohlhammer.

Lenhard, W., \& Schneider, W. (2006). ELFE 1-6. Ein Leseverständnistest für Erst- bis Sechstklässler. Göttingen: Hogrefe.

McElvany, N., Becker, M., \& Lüdtke, O. (2009). Die Bedeutung familiärer Merkmale für Lesekompetenz, Wortschatz, Lesemotivation und Leseverhalten. Zeitschrift für Entwicklungspsychologie und Pädagogische Psychologie, 41, 121-131.

Melby-Lervåg, M., \& Lervåg, A. (2014). Reading comprehension and its underlying components in second-language learners: a meta-analysis of studies comparing first- and second-language learners. Psychological Bulletin, 140, 409-433.

Naumann, J., Artelt, C., Schneider, W., \& Stanat, P. (2010). Lesekompetenz von PISA 2000 bis PISA 2009. In E. Klieme, C. Artelt, J. Hartig, N. Jude, O. Köller, M. Prenzel, W. Schneider \& P. Stanat (Hrsg.), PISA 2009. Bilanz nach einem Jahrzehnt (S. 23-71). Münster: Waxmann.

Netten, A., Droop, M., \& Verhoeven, L. (2011). Predictors of reading literacy for first and second language learners. Reading and Writing, 24, 413-425.

Netten, A., Voeten, M., Droop, M., \& Verhoeven, L. (2014). Sociocultural and educational factors for reading literacy decline in the Netherlands in the past decade. Learning and Individual Differences, $32,9-18$. 
Niklas, F., Schmiedeler, S., \& Schneider, W. (2010). Heterogenität in den Lernvoraussetzungen von Vorschulkindern. Zeitschrift für Grundschulforschung, 3(1), 18-31.

Paetsch, J., Felbrich, A., \& Stanat, P. (2015). Der Zusammenhang von sprachlichen und mathematischen Kompetenzen bei Kindern mit Deutsch als Zweitsprache. Zeitschrift für Pädagogische Psychologie, 29(1), 19-29.

Pareiss, M., \& Schwandtner, U. (2013). Schüler/innen mit Migrationshintergrund. In U. Schwandtner, B. Toferer \& C. Schreiner (Hrsg.), PISA 2012. Internationaler Vergleich von Schülerleistungen. Erste Ergebnisse Mathematik, Lesen, Naturwissenschaft (S. 48-49). Graz: Leykam.

Perfetti, C. A., Landi, N., \& Oakhill, J. (2005). The acquisition of reading comprehension skill. In M. J. Snowling \& C. Hulme (Hrsg.), The science of reading: a handbook (S. 227-247). Malden, MA: Blackwell Publishing.

Richter, M. (2008). Tabakkonsum im Jugendalter zwischen sozialer Herkunft, Gleichaltrigengruppe und Schule. In A. Groenemeyer \& S. Wieseler (Hrsg.), Soziologie sozialer Probleme und sozialer Kontrolle. Realitäten, Repräsentationen und Politik (S. 375-394). Wiesbaden: VS Verlag für Sozialwissenschaften.

Salchegger, S., Herzog-Punzenberger, B., \& Filzmoser, S. (2012). Migrationshintergrund und Lesekompetenz: Entwicklung seit dem Jahr 2000. In B. Suchán, C. Wallner-Paschon \& C. Schreiner (Hrsg.), PIRLS \& TIMSS 2011. Die Kompetenzen in Lesen, Mathematik und Naturwissenschaft am Ende der Volksschule. Österreichischer Expertenbericht (S. 67-84). Graz: Leykam.

Salchegger, S., Wallner-Paschon, C., Schmich, J., \& Höller, I. (2016). Kompetenzentwicklung im Kontext individueller, schulischer und familiärer Faktoren. In B. Suchán \& S. Breit (Hrsg.), PISA 2015. Grundkompetenzen am Ende der Pflichtschulzeit im internationalen Vergleich (S. 77-99). Graz: Leykam.

Schnepf, S. V. (2007). Immigrants' educational disadvantage: an examination across ten countries and three surveys. Journal of Population Economics, 20, 527-545.

Schreiner, C. (2012). Bildung der Eltern und Schülerleistungen in Österreich. In B. Suchán, C. WallnerPaschon, S. Bergmüller \& C. Schreiner (Hrsg.), PIRLS \& TIMSS 2011. Schülerleistungen in Lesen, Mathematik und Naturwissenschaft in der Grundschule. Erste Ergebnisse (S. 48-49). Graz: Leykam.

Sirin, S. (2005). Socioeconomic status and academic achievement: a meta-analytic review of research. Review of Educational Research, 75, 417-453.

Suchán, B. (2012). Lesekompetenz im Ländervergleich. In B. Suchán, C. Wallner-Paschon, S. Bergmüller \& C. Schreiner (Hrsg.), PIRLS \& TIMSS 2011. Schülerleistungen in Lesen, Mathematik und Naturwissenschaft in der Grundschule. Erste Ergebnisse (S. 12-13). Graz: Leykam.

Suchán, B., Wallner-Paschon, C., Bergmüller, S., \& Schreiner, C. (Hrsg.). (2012). PIRLS \& TIMSS 2011. Schülerleistungen in Lesen, Mathematik und Naturwissenschaft in der Grundschule. Erste Ergebnisse. Graz: Leykam.

Toferer, B., Höller, I., Schmich, J., \& Suchán, B. (2016). Kompetenzen der Schüler/innen in Lesen. In B. Suchán \& S. Breit (Hrsg.), PISA 2015. Grundkompetenzen am Ende der Pflichtschulzeit im internationalen Vergleich (S. 57-64). Graz: Leykam.

Vogtenhuber, S., Siegle, T., \& Lassnigg, L. (2016). Indikatoren A: Kontext des Schul- und Bildungswesens. In M. Bruneforth, L. Lassnigg, S. Vogtenhuber, C. Schreiner \& S. Breit (Hrsg.), Das Schulsystem im Spiegel von Daten und Indikatoren. Nationaler Bildungsbericht Österreich 2015, (Bd. 1, S. 21-35). Graz: Leykam

Wallner-Paschon, Ch (2012). Leseleistungen in den Teilbereichen „Verstehensprozesse“. In B. Suchán, C. Wallner-Paschon, S. Bergmüller \& C. Schreiner (Hrsg.), PIRLS \& TIMSS 2011. Schülerleistungen in Lesen, Mathematik und Naturwissenschaft in der Grundschule. Erste Ergebnisse (S. 22-23). Graz: Leykam.

Wang, M., Haertel, G., \& Walberg, H. (1993). Toward a knowledge base for school learning. Review of Educational Research, 63, 249-294.

Zöller, J., \& Roos, J. (2009). Einfluss individueller Merkmale und familiärer Faktoren auf den Schriftspracherwerb. In J. Roos \& H. Schöler (Hrsg.), Entwicklung des Schriftspracherwerbs in der Grundschule. Längsschnittanalyse zweier Kohorten über die Grundschulzeit (S. 47-107). Wiesbaden: VS Verlag für Sozialwissenschaften. 